\title{
Compact, Energy EFFICIENT Neutron Source: Enabling Technology for Various Applications
}

A. Hershcovitch, T. Roser

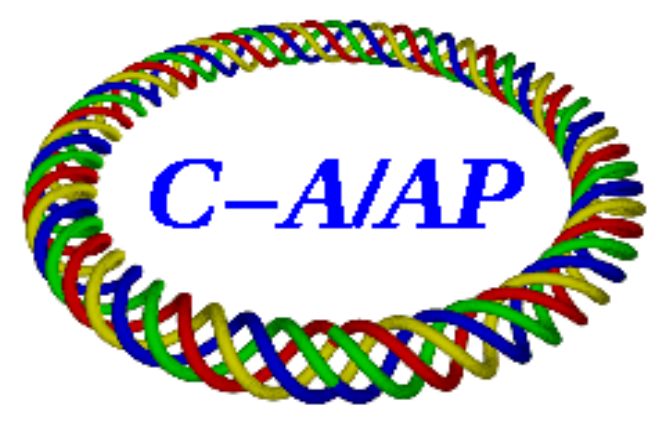

\section{Collider-Accelerator Department Brookhaven National Laboratory Upton, NY 11973}

Notice: This document has been authorized by employees of Brookhaven Science Associates, LLC under Contract No. DE-AC02-98CH10886 with the U.S. Department of Energy. The United States Government retains a nonexclusive, paid-up, irrevocable, world-wide license to publish or reproduce the published form of this document, or allow others to do so, for United States Government purposes. 


\title{
Compact, Energy EFFICIENT Neutron Source: Enabling Technology for Various Applications
}

\author{
Ady Hershcovitch and Thomas Roser
}

A novel neutron source comprising of a deuterium beam (energy of about $100 \mathrm{KeV}$ ) injected into a tube filled with tritium gas and/or tritium plasma that generates D-T fusion reactions, whose products are $14.06 \mathrm{MeV}$ neutrons and $3.52 \mathrm{MeV}$ alpha particles, is described. At the opposite end of the tube, the energy of deuterium ions that did not interact is recovered. Beryllium walls of proper thickness can be utilized to absorb 14 $\mathrm{MeV}$ neutrons and release $2-3$ low energy neutrons. Each ion source and tube forms a module. Larger systems can be formed from multiple units. Unlike currently proposed methods, where accelerator-based neutron sources are very expensive, large, and require large amounts of power for operation, this neutron source is compact, inexpensive, easy to test and to scale up. Among possible applications for this neutron source concept are sub-critical nuclear breeder reactors and transmutation of radioactive waste.

\section{Introduction}

Presently utilized and previously proposed large flux neutron sources are either nuclear reactor based or accelerator based. Both methods have major shortcomings: the first method generates radioactive waste and has high proliferation risk. Presently operating spallation neutron sources as well as previously proposed accelerator based neutron sources $^{1-5}$, for sub-critical nuclear reactors are complex, expensive and require large amounts of power input to be sustained. Consequently, none of the previously proposed accelerator based neutron sources for sub-critical nuclear reactors and/or accelerator transmutation of radioactive waste have been implemented. Additionally, these accelerators are very large in size, a fact that makes any experimental testing very expensive. For sake of completeness, it should be mentioned that various hybrids, symbiotic and Augean fission-fusion schemes ${ }^{6}$ (for critical reactors) have been proposed as mostly an intermediate step towards fusion power. Arguments were invoked that in a hybrid or in a symbiotic system, the cost per neutron will be cheaper than in fission reactors. Therefore, breeding will be cheaper than in a fission breeder. But, the fusion part of the reactor, in addition to its complexity would have siphoned 100's of MW of electrical power from the fission part of the reactor. In this paper a novel, relatively simple method for neutron generation is described. Among possible applications for this neutron source are thorium breeders, sub-critical nuclear breeder reactors and transmutation of radioactive waste.

Though the method can have a few variations, the basic concept comprises of a DC deuterium beam (energy of about $100 \mathrm{KeV}$ ), which is to be injected into a tube filled with tritium gas or tritium plasma to generate D-T fusion reactions whose products are 14.06 $\mathrm{MeV}$ neutrons and $3.52 \mathrm{MeV}$ alpha particles. At the opposite end of the tube the energy of deuterium ions that did not interact is recovered. Each ion source and tube forms a module. Larger systems can be formed from multiple units. A number of embodiments of steady state beams striking gas and/or plasma targets are considered. At first glance, a DC 
beam striking a gas and/or plasma targets is not attractive, since the ion beam energy is attenuated at a faster rate than the fusion energy produced. To overcome beam attenuation, self-pinched beam propagation and other enhanced beam propagation phenomena, which were observed in electrons launched into atmosphere through Plasma Windows, could be used to mitigate beam attenuation. But, this scenario carries high risk, since it is based on an observed but not understood electron beam phenomena, which, in addition, has not yet been observed with ion beams. However, as an ion beam heats the target gas a plasma, which is needed to ensure beam propagation, is generated. Regardless, energy recovery should reduce energy loss by about a factor of 3 .

A safer scenario is to inject the ion beams into thin tritium plasma targets with hot electrons, where attenuation is greatly reduced. Beam propagation can be further enhanced with vortex stabilized discharges as shown in figures 1,2 , and 3 , or with magnetic fields (magnetized plasma target as shown in figures 6 and 7). Variation on this technique could be a pure ion target, where ions are confined in a gated ion (Penning like) trap and space charge neutralized by a counterstreaming (with respect to the deuterium) electron beam.

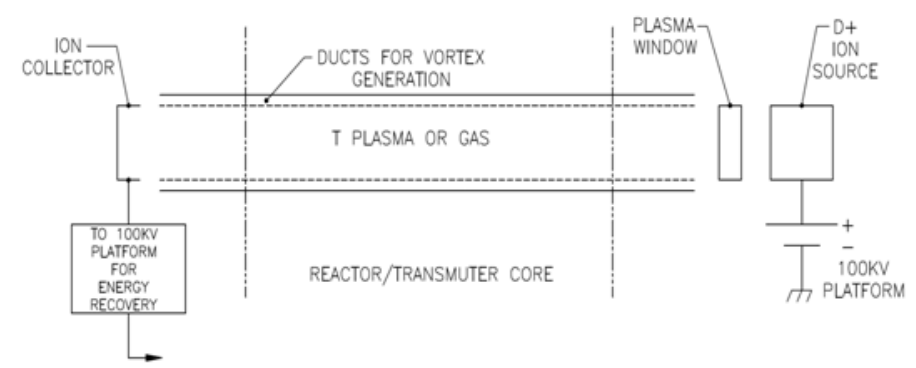

Figure 1 Deuterium beam injected horizontally through tritium plasma or gas target, confined by vortex stabilized plasma, towards a collector for energy recovery.

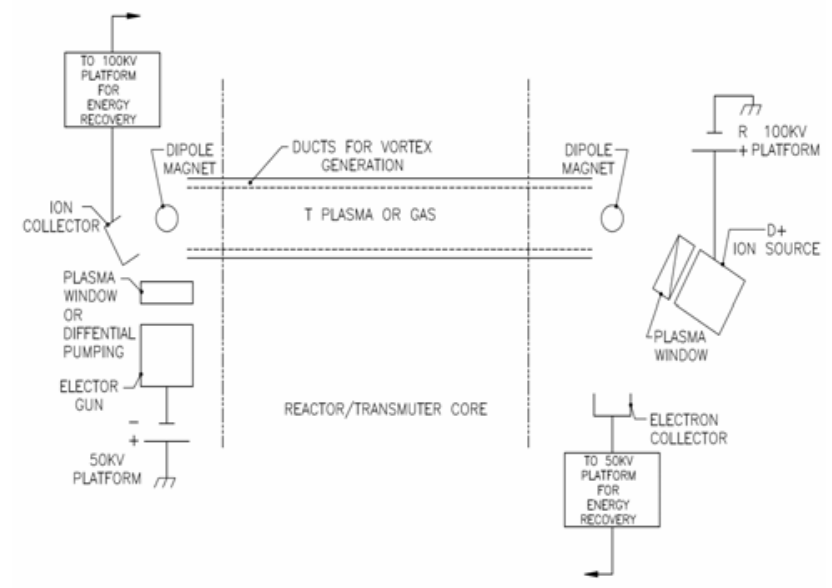

Figure 2 Deuterium beam injected horizontally through tritium plasma or gas target, confined by vortex stabilized plasma and/or electron beams, towards a collector for energy recovery. 


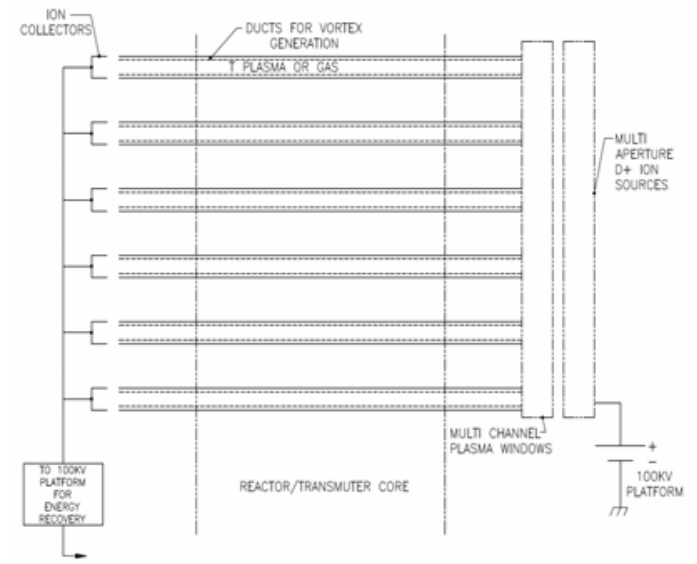

Figure 3 Figure 1 embodiment showing multiple horizontally injected deuterium beams.

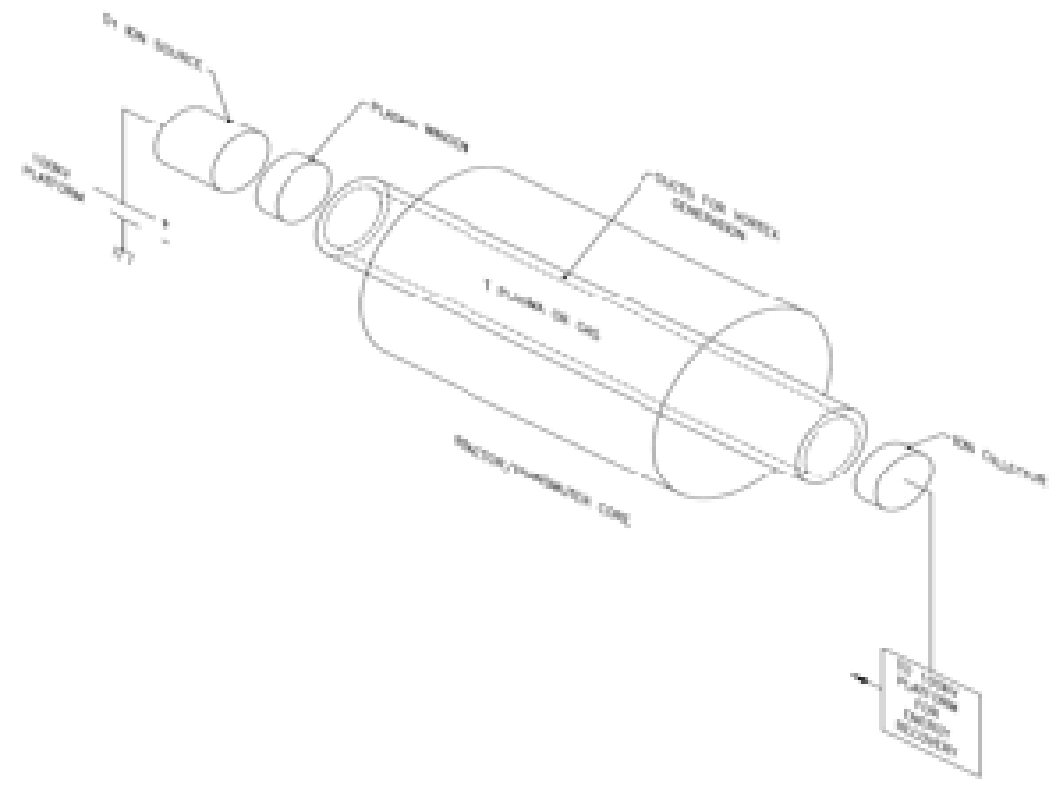

Figure 4 Deuterium beam injected vertically through tritium plasma or gas target, confined by vortex stabilized plasma, towards a collector for energy recovery.

\section{Fusion Power Generation and neutron source}

Considering a $100 \mathrm{KeV}$ deuterium beam of current $\mathrm{I}_{\mathrm{d}}$, entering a tritium gas target with an atomic density $n_{t}$. The deuterium-tritium ion fusion power $P_{f}$ generated after the beam travels a distance $\mathrm{x}$ is

$\mathrm{P}_{\mathrm{f}}=\boldsymbol{I}_{\mathbf{d}}\left(1-\exp \left\{-\left[\mathrm{n}_{\mathrm{t}} \sigma \mathrm{x}\right]\right\}\right) \mathrm{Q} \approx \boldsymbol{I}_{\mathbf{d}} \mathrm{Q} \mathrm{n}_{\mathrm{t}} \sigma \mathrm{x}$

where $\sigma$ is the D-T fusion cross section, $\boldsymbol{I}_{\boldsymbol{d}}=\mathrm{I}_{\mathrm{d}} / 1.6 \times 10^{-19} \mathrm{C}$ (i.e. particle current), $\mathrm{x}$ is the interaction length. The energy generated per reaction $\mathrm{Q}=17.58 \mathrm{MeV} /$ fusion reaction and $\mathrm{n}_{\mathrm{t}}$ is the number of triton per $\mathrm{cm}^{3}$.

Evaluating equation 1 can be straight forward within an energy range, if deuterium beam does not slow down significantly over the interaction region. The cross section $\sigma$ is well 
known. It has a broad peak ${ }^{7}$ of 6 barns in the energy range of about $75-125 \mathrm{KeV}$ (please see figure 2.2 in reference 7 ).

The total power lost due to the interaction of the deuterons with the electrons of the tritium gas, either by ionization or scattering, is given by the stopping power $\mathrm{dE} / \mathrm{dx}$ of deuteron in hydrogen times the number of electrons per $\mathrm{cm}^{3}$ :

$\mathrm{P}_{\text {loss }}=\boldsymbol{I}_{\mathbf{d}}\left(1-\exp \left\{-\left[\mathrm{dE} / \mathrm{dx} \mathrm{n}_{\mathrm{e}} \mathrm{x}\right]\right\}\right) \mathrm{Q} \approx \boldsymbol{I}_{\mathbf{d}} \mathrm{dE} / \mathrm{dx} \mathrm{n}_{\mathrm{e}} \mathrm{X}$.

The ratio of fusion power to the lost power is then:

$\mathrm{P}_{\mathrm{f}} / \mathrm{P}_{\text {loss }}=\left(\mathrm{Q} \mathrm{n}_{\mathrm{t}} \sigma\right) /\left(\mathrm{dE} / \mathrm{dx} \mathrm{n}_{\mathrm{e}}\right)$

Or for a neutral tritium gas where $n_{t}=n_{e}$

$\mathrm{P}_{\mathrm{f}} / \mathrm{P}_{\text {loss }}=(\mathrm{Q} \sigma) /(\mathrm{dE} / \mathrm{dx})$.

For an average deuteron energy of $100 \mathrm{keV}$ we have: $\mathrm{Q} \approx 17.6 \mathrm{MeV}, \sigma \approx 6 \times 10^{-24} \mathrm{~cm}^{2}$, and $\mathrm{dE} / \mathrm{dx} \approx 4.5 \times 10^{-21} \mathrm{MeV} \mathrm{cm}^{2}$ and then

$\mathrm{P}_{\mathrm{f}} / \mathrm{P}_{\text {loss }}=0.023$.

This simple single particle calculation gives a rather low efficiency of $2.3 \%$. Similarly the neutron flux per lost beam power is:

$\mathrm{dn} / \mathrm{dt} / \mathrm{P}_{\text {loss }}=\mathrm{n} / \Delta \mathrm{E}=\sigma /(\mathrm{dE} / \mathrm{dx})=0.0013 \mathrm{MeV}^{-1}=0.8 \times 10^{10}$ neutrons $/ \mathrm{sec} /$ Watt

Note that the interaction with the electrons can be substantially suppressed as discussed below. For example, the propagation of $100 \mathrm{keV}$ electrons in air was shown to be about 10 times more than expected from the stopping power. A similarly reduced effective stopping power for $100 \mathrm{keV}$ deuteron in tritium gas would increase the efficiency to $23 \%$ and the neutron flux would increase to $0.013 \mathrm{MeV}^{-1}$ or $0.8 \times 10^{11}$ neutrons/sec/Watt.

\section{Analysis of the High Gas Density Scenario; Comparison to the GA concept}

Computing the range of $100 \mathrm{KeV}$ deuterium beam, however, is rather tricky. To begin with, range, which can be extracted from available literature is basically for single particles and not beams. As an ion passes through matter, its slowing down is best described by the Bethe-Bloch equation ${ }^{8}$. However, at energies below $6 \mathrm{GeV}$, various corrections ${ }^{9}$, which reflect the multiple scattering of the attenuation process, are required. CSDA (continuous-slowing-down approximation) is used to compute range. For electrons, protons and alpha particles range has been computed ${ }^{10}$. Closest to our case of interest is protons in hydrogen, which is easily converted to deuterium in tritium (100 $\mathrm{KeV}$ deuteriums are equivalent to $50 \mathrm{KeV}$ protons). At $50 \mathrm{KeV}$ CSDA range ${ }^{10}$ (can be found at the NIST website) is $1.85 \times 10^{-5} \mathrm{~g} / \mathrm{cm}^{2}$. Since hydrogen has a density (at 
atmospheric pressure and room temperature) of $8.3 \times 10^{-5} \mathrm{~g} / \mathrm{cm}^{3}$, the CSDA range is about $0.22 \mathrm{~cm}$. Having hydrogen as target is identical to having tritium, since practically all particle slowing down and scattering is at the atomic level at these energies.

But, this CSDA range is smaller than the fusion interaction length for the following reasons: first, the CSDA is a penetration length. At energies below $6 \mathrm{GeV}$, particle attenuation process is dominated by multiple small angle scattering ${ }^{9}$. Thus, a particle travels a path, which is larger than the range (and so is the fusion interaction length) due to this random walk process. Second, intense particle beams have substantially longer ranges than single particles of the same energy due to various collective effects. For example, $100 \mathrm{KeV}$ electrons have a CSDA range ${ }^{10}$ in dry air of $13 \mathrm{~cm}$ (dry range $1.6 \times 10^{-2}$ $\mathrm{g} / \mathrm{cm}^{2}$, and dry air density near sea level is $\left.1.2 \times 10^{-3} \mathrm{~g} / \mathrm{cm}^{3}\right)$. However, electrons from 100 $\mathrm{KeV}$ electron beam of only a few $\mathrm{mA}$, which enter atmosphere, can be observed well over a meter down stream.

Since CSDA gives a range of $0.22 \mathrm{~cm}$, it's likely that the range of a beam could be as high as $2 \mathrm{~cm}$. Therefore, it's reasonable to assume the fusion interaction length $\mathrm{x}$ to be about $2 \mathrm{~cm}$. Equation 1 can now be used to estimate power generated by $100 \mathrm{KeV}(\sigma=6$ barns) 1-Ampere, of deuterium striking a 1 atmosphere tritium gas target. 1-Ampere of deuterium implies $6.25 \times 10^{18}$ deuterium ions/second; therefore equation 1 yields $11.5 \mathrm{~kW}$ (atomic tritium density must be used, since [unlike scattering] fusion reaction occurs at the nuclear level). Thus, the simple scenario of a DC beam striking a room-temperature gas target at atmospheric pressure can generate fusion power that is only $11.5 \%$ of the beam power. The problem is that the ion beam is attenuated faster than fusion reaction rate by a factor that is larger than energy amplification (fusion energy/beam particle energy). That's thermal fusion energy power (another factor of 3 is lost in thermal to electrical power conversion). If tritium target thickness is programmed to prevent deuterium slowing down below $75 \mathrm{KeV}$, substantial energy can be recovered, hence a factor of 2.5 can be gained (for $125 \mathrm{KeV}$ deuterium in a tritium gas target, slow down to $75 \mathrm{KeV}$ ), thus increasing the fusion power to $23 \%$ of deuterium beam power loss. Under this scenario, about $4 \times 10^{15}$ neutrons/second (fusion power $/ 2.8 \times 10^{-12}$ Joule/reaction) can be generated at a deuterium power loss of $38.5 \mathrm{~kW}(50 \mathrm{~kW}-11.5 \mathrm{~kW}$ fusion power generated). Therefore, $1 \times 10^{11}$ neutrons/sec/Watt are generated.

Comparison can now be made between this neutron generation rate and that of the GA concept ${ }^{5}$, which is considered to be the most efficient scheme to date. The GA concept ${ }^{5}$ is a spallation neutron source for transmutation of nuclear waste, which is based on $1 \mathrm{GeV}$ $15 \mathrm{~mA}$ proton beam, i.e. $15 \mathrm{MW}$ of incident proton beam power! This spallation process generates 40 useful neutrons/proton. Thus, the GA concept is designed to generate $3.75 \times 10^{18}$ neutrons/sec. Hence it could generate $2.5 \times 10^{11}$ neutrons $/ \mathrm{sec} /$ Watt of proton beam power. But, RF acceleration is only $10-20 \%$. This factor alone reduces power cost of neutron generation to $3.75 \times 10^{10}$ neutrons/sec/Watt $(15 \%$ acceleration efficiency). Superconducting cavities can reach $80 \%$ but require large refrigeration with power efficiency of no more than $50 \%$. Hence, the use of superconducting cavities increases the power efficiency to the scenario presented thus far. This comparison does not even address the difference in complexities and cost. Based on the SNS experience, a 
spallation neutron source is a major project. SNS, whose cost reached billions of dollars, has not yet reached peak performance goals.

Higher beam energies have no advantage as both scattering and fusion cross-sections decline at roughly the same rate. Additionally, for multi-MeV beams point $\mathrm{RF}$ acceleration (low efficiency) is needed. Other power deficient schemes with high-energy beams have already been proposed ${ }^{1-5}$. Schemes involving beams to drive old fusion devices (called the Wet Wood Burner) were examined extensively ${ }^{1-14}$ in the 1970's. But, those were plagued by some of ion beam is attenuation problems as well other issues that the fusion community is trying to solve.

Our assumption of interaction length is justified, since there are alternative options for extending the fusion interaction length like enhancement of ion beam propagation by plasma effects like self-pinched propagation. Proof exists ${ }^{15}$ for self-pinched propagation of $\mathrm{kA}, \mathrm{MeV}$ electron beams in atmospheric pressure, as well as a $400 \mathrm{kA}, 1.2 \mathrm{MeV}$ proton beam propagation ${ }^{16}$ in a lower pressure. Evidence for enhanced charged particle beam propagation is further described in the next paragraphs.

Any beam of propagating charged particles generates an azimuthal magnetic field. This magnetic field causes the beam to pinch, i.e. there is an inward $\mathbf{J x B}$ force due to the Lorentz force. In self-pinched propagation, the self-magnetic pinch force balances the transverse pressure. Although it was observed in $\mathrm{MeV}, \mathrm{kA}$ beams, it is a function of the current density ${ }^{15}$; hence, it can occur in charged particle beams with lower current and energy. Another factor that enables propagation is return current inside the plasma generated by propagating beam ionization. This return current increases conductivity ${ }^{15,16}$ for the propagating beam. Current carrying plasma channels act as lenses due to the $\mathbf{J x B}$ force. With proper orientation, $\mathbf{J} \mathbf{x B}$ force can be inward, i.e. focusing. Plasma channels have been used to propagate ${ }^{17-19}$ a variety of light ion beams for distances of up to 5 meters.

Unlike the above results with pulse, $\mathrm{kA}, \mathrm{MeV}$ beams, there is reasonably strong evidence that self-pinched propagation was achieved with $6-15 \mathrm{~mA}, 90-150 \mathrm{KeV}$ electron beams $^{20,21}$ after these beams traveled through a Plasma Window ${ }^{22-25}$ from vacuum to atmosphere. The Plasma Window is a novel apparatus that utilizes a stabilized plasma arc as an interface between vacuum and atmosphere or pressurized targets without solid material. In addition to sustaining a vacuum atmosphere interface, the plasma has a lensing effect on charged particles. The plasma current generates an azimuthal magnetic field, which exerts a radial Lorentz force on charged particles moving parallel to the current channel. With proper orientation of the current direction, the Lorentz force is radially inward. This feature can be used to focus beams to very small spot size and to overcome beam dispersion due to scattering by atmospheric atoms and molecules. Earlier results have been the following: Vacuum (pressure of $\sim 10^{-6}$ Torr) was successfully separated from atmosphere and from a gas target pressurized up to 9 bar $^{26}$. A $2 \mathrm{MeV}$ proton beam was propagated from vacuum through the plasma window into atmospheric pressure with no measurable energy $\operatorname{loss}^{27}$ or beam degradation. 
Recent results ${ }^{28}$ strongly suggest that low energy electron beams (from an electron beam welder) propagate in atmosphere much further when a Plasma Shield is generated from a plasma window to a target object than without the plasma shield. Plasma Shield ${ }^{28}$ is designed to chemically and thermally shield a target object by engulfing an area subjected to beam treatment with inert plasma. The shield consists of a vortex-stabilized arc that is employed to shield beams and workpiece area of interaction from atmospheric or liquid environment.

Purpose of the above discussion was to show that a collimated beam has a longer range than what CSDA predicts for single particles. And, that it is possible to use various techniques including but not limited to plasma windows, vortex stabilized arcs and electron beams to generate azimuthal magnetic field and return current to enhance deuterium ion beam propagation. Air-boring, i.e. gas rarifying is unlikely to occur, since the deuterium ion current is low (only $1 \mathrm{~A}$ ).

\section{Analysis of Low Density Scenarios}

Computing energy loss of $100 \mathrm{KeV}$ deuterium beam propagating through a low density tritium target can be relatively straight forward (within certain ranges of parameters). Low density refers to cases where deuterium beam range is larger than any envisioned apparatus, i.e., the deuterium beam slows down at the end of a reactor or transmuter core to no less than $75 \mathrm{KeV}$. In these cases, deuterium ion energy loss is dominated by ions slowing down due to collisions with plasma and/or gas particles. Three low density targets scenarios are considered:

1. A deuterium beam in tritium gas.

2. A deuterium beam in tritium plasma with low temperature electrons.

3. And, a deuterium beam in tritium plasma with high temperature electrons.

Ion beams moving through highly ionized plasmas are predominantly slowed down by dynamic friction" with slower moving electrons in the case of "cold" target plasma. Dynamic friction is particularly potent when the "fast" ion beam velocity is close to the speed associated with plasma electrons. Therefore, it is critical to have a velocity difference between the species as large as possible to minimize ion slowing down, especially in the case of slow electrons. Thus, "high" and "low" temperature electrons are for cases where electrons are faster, or slower than the deuterium ions.

However in cases of relatively large target thickness confined plasmas, deuterium power deposition can significantly increase plasma temperature, and consequently affect deuterium beam slow-down. Even though it complicates calculations, the result is a more appealing concept.

\section{IVa Deuterium Beam in Tritium Gas}

In the high density case, the tritium target is short (only $2 \mathrm{~cm}$ long). By extending the target length to the size of a reactor or transmuter core while reducing the tritium density proportionally, technical simplification can be accomplished. Though, in some designs 
point-like neutron sources might be preferential. Nevertheless, energy analysis as well as all other results can be extrapolated from the previous section.

For deuterium in tritium plasmas, two cases are considered: low electron temperature, where deuteron ions are faster than plasma electrons, and high electron temperature, where deuterium ions are slower than plasma electrons.

Computing deuterium ion slowing down and energy loss in plasma can be performed using the test particle model, which was originated by Norman Rostoker ${ }^{29}$. In the cases of very fast or slow test particle, straightforward formulas are available $\mathrm{e}^{30}$.

\section{$\underline{\mathrm{IVb}}$ Deuterium Beam in Cold Electron Tritium Plasma}

Next to be analyzed is a case of deuterium ions in $100 \%$ ionized plasma like from a hollow cathode $\operatorname{arc}^{31-36}$ with temperatures in the range of $12-15 \mathrm{eV}$, and densities exceeding $1 \times 10^{14} \mathrm{~cm}^{-3}$. Confinement and stabilization of this type of target plasma can be accomplished by schemes shown in figures 1-4 involving vortices and possibly electron beams; or, magnetic confinement as shown in figure 5 .

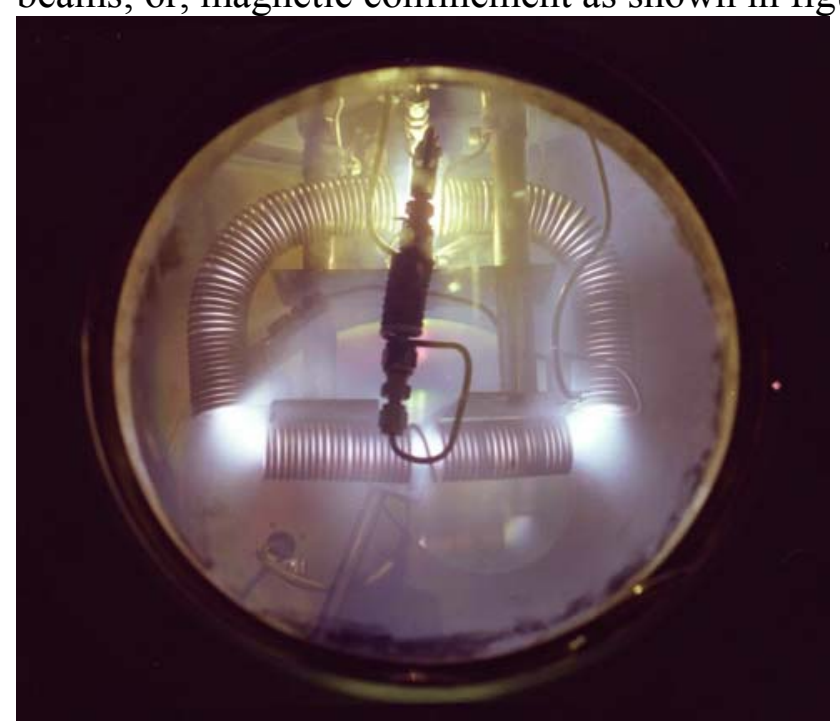

Figure 5 photo of reference 33 plasma target confined by closed loop D-shaped solenoidal magnetic field.

A possible magnetic confinement of target plasma is shown in figures 8 and 9. Both involve plasma targets described in references 33 and 36.

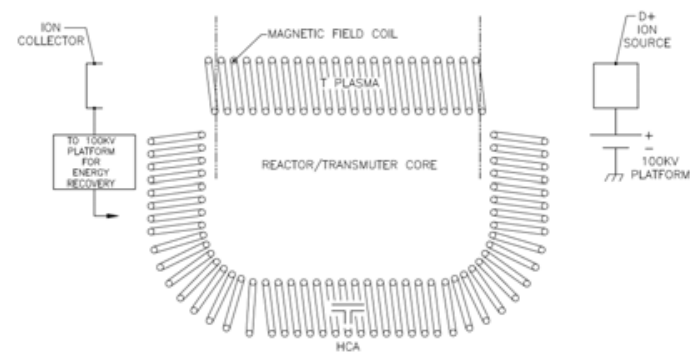

Figure 6 scheme involving magnetized target plasma. 


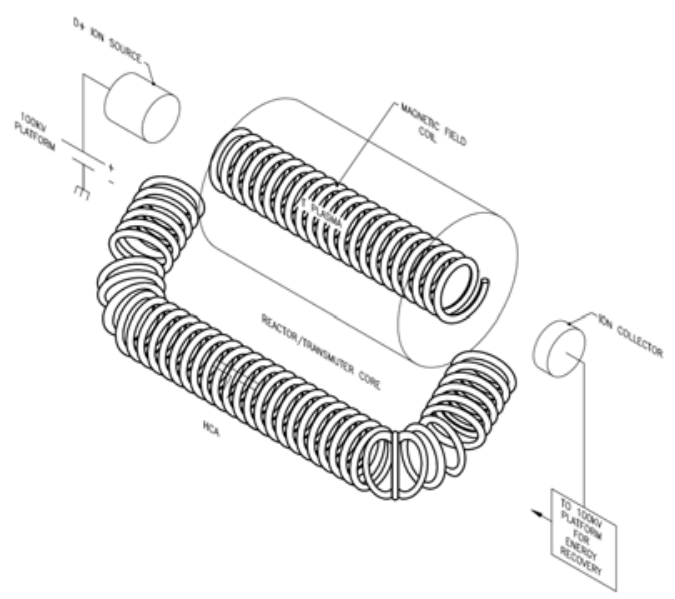

Figure 73 -D display of the figure 6 scheme.

Electron thermal velocity and ion beam velocity are given by ${ }^{30}$

$V_{\mathrm{Te}}=4.19 \times 10^{7} \mathrm{~T}_{e}^{1 / 2} \mathrm{~cm} / \mathrm{sec}$

and

$V_{i}=1.38 \times 10^{6} \mu^{-1 / 2} E_{i}^{1 / 2} \mathrm{~cm} / \mathrm{sec}$

respectively, where $\mu$ is ion mass expressed in units of proton mass $\mathrm{T}$ and $\mathrm{E}$ are in $\mathrm{eV}$. From equations 2 and $3,15 \mathrm{eV}$ thermal electrons have a velocity of $1.6 \times 10^{8} \mathrm{~cm} / \mathrm{sec}$, while $100 \mathrm{KeV}$ deuterons have a velocity of $3 \times 10^{8} \mathrm{~cm} / \mathrm{sec}$. Ion energy loss, due to slowing down by electrons (slowing down on ions is over 3 orders of magnitude lower), rate $v^{\mathrm{i} / \mathrm{e}}$ is defined as

$$
d E_{i} / d t=-v^{i / e} E_{i}
$$

It's basically twice the slowing down rate, from which parallel and perpendicular diffusion rates in velocity space are subtracted, which in this case it is close to the slowing down rate. If there is relatively little change in ion beam velocity Equation 4 can be written as

$$
\Delta E_{i} / \Delta t \approx-v^{i / e} E_{i}, \text { hence } \Delta E_{i} \approx-v^{i / e} E_{i} \Delta t
$$

For ions faster than thermal electrons $v^{\mathrm{i} / \mathrm{e}}\left(\mathrm{in} \sec ^{-1}\right)$ is given by ${ }^{30}$

$v^{i / e}=n_{e} Z^{2} \lambda_{i e} 1.7 \times 10^{-4} \mu^{1 / 2} E_{i}^{-3 / 2}$ 
where $\mathrm{n}$ is density in $\mathrm{cm}^{-3}, \lambda$ is Coulomb logarithm, which in this case is about 10 (from the NRL formulary), and $\mathrm{Z}$ is ion charge state. Unless otherwise noted all other units are cgs, $\mathrm{E}$ and $\mathrm{T}$ are in $\mathrm{eV}$.

For plasma density of $3 \times 10^{14} \mathrm{~cm}^{-3}$, e.g. $v^{\mathrm{i} / \mathrm{e}}$ is $2.28 \times 10^{4} \mathrm{sec}^{-1}$. Although energy deposition rate from deuterium beam to tritium ions is about 4 orders of magnitude lower, energy equilibration rate between plasma electrons and tritons is rather fast. That rate is given by $^{30}$

$v_{\varepsilon}^{e / i}=3.2 \times 10^{-9} n_{e} Z^{2} \lambda \mu^{-1} T_{e}^{-3 / 2}$

In this case of $15 \mathrm{eV}$ plasma temperature, $v_{\varepsilon}^{e / i}=5.5 \times 10^{4} \mathrm{sec}^{-1}$, i.e., close to twice the deuteron electron energy transfer rate. Therefore, significant energy is transferred to the tritium ions. Nevertheless, it's important to note that as the electron temperature increase, the rate of tritium heating decreases. Additionally, as it becomes apparent in the next subsection, beam energy loss drop drastically, when electron thermal speed exceeds ion beam velocity. Therefore, the electron temperature strongly affects neutron generation power cost, i.e. neutrons/sec/Watt of deuterium power loss.

A major difficulty in evaluating this scenario is determination of equilibrium electron temperature of magnetically confined plasma targets ${ }^{33,36}$ that are heated by an ion beam, for which no beam heating data is available (in reference 36 e.g. $8 \mathrm{MeV}$ beams with currents of only about a $\mu \mathrm{A}$ were used). One possibility is to estimate various plasma energy loss mechanisms and compare them to deuterium energy deposition. Since some data exists $^{31-36}$ for particle and energy loss in meter long hollow cathode arc generated plasma targets, estimation of the equilibrium electron temperature range is made before computing power cost of neutron generation. Obviously equilibrium temperature is reached when target plasma power loss equals to target heating by the deuterium ion beam, which can be calculated from equations 5 and 6 . Therefore based on these equations, in 1-meter travel each deuteron would deposit a little less than $760 \mathrm{eV}$ in plasma electrons, part of which is then deposited in plasma ions (it takes $3.3 \times 10^{-7}$ second for a deuterium beam to travel one meter). At low electron temperatures, close to half of that power will be quickly transferred to the tritium ions.

Power range loss is estimated from some empirical data (or from Bohm diffusion) and from radiation calculations. In this case of a simple cylindrical geometry, particle and energy confinement times are most likely extremely close, if not identical. Thus, a reasonable estimate of power can be attained from the product of temperature and particle loss rate; or, use known plasma target generation power supply requirements and subtract ionization energy (i.e. power required for ionizing the original plasma, which does not factor in beam power deposition/loss balance; equivalent to filament power in other schemes) and radiation losses. BNL experimental data is available for 1 inch-diameter magnetized plasma ${ }^{33,36}$ for open geometry solenoidal plasma ${ }^{36}$ and for a closed loop Dshaped solenoidal magnetic field ${ }^{33}$. In these experiments, like in many hollow cathode arcs, plasma densities of $2 \times 10^{14} \mathrm{~cm}^{-3}$ have been routinely reached. In the 1 -meter long open geometry case, gas flow rate was 0.5 Torr-litter/second, which means that $1.8 \times 10^{19}$ 
hydrogen molecules per second are injected to sustain the arc. If the tritium plasma density is "pushed" to $3 \times 10^{14} \mathrm{~cm}^{-3}$, that number will probably become $2.7 \times 10^{19}$ tritium molecules per second. Therefore, power loss is likely to be $2.7 \times 10^{19} \times \mathrm{T} \times 1.6 \times 10^{-19}=4.32 \mathrm{~T}$ Watt (plasma temperature $\mathrm{T}$ in $\mathrm{eV}$ ). In the case of closed loop D-shaped solenoidal magnetized plasma, gas flow rate was 0.06 Torr-litter/second, which for plasma density of $3 \times 10^{14} \mathrm{~cm}^{-3}$ that number will probably become $3.24 \times 10^{18}$ tritium molecules per second. And, power loss is likely to be $0.52 \mathrm{~T}$ Watt (most likely does not scale as T). In that case total plasma length was about $75 \mathrm{~cm}$. But, power loss, which most likely dominated by losses in the large magnetic field gap would not change much, if the plasma length were to be increased to 1 meter (it might have increased to $0.6 \mathrm{~T}$ Watt). Bremsstrahlung is given by ${ }^{7,30}$ (in Watt $\mathrm{cm}^{-3}$; $\mathrm{T}$ in $\mathrm{eV}$ )

$P_{B r}=1.69 \times 10^{-32} n^{2} T_{e}^{1 / 2}=1.52 \times 10^{-3} T_{e}^{1 / 2}{\mathrm{Watt} \mathrm{cm}^{-3}}^{1 / 2}$

For 1-inch diameter plasma equation 8 , becomes

$P_{B r}=0.77 T_{e}^{1 / 2}$ Watt/meter of tritium target length

Cyclotron radiation is orders of magnitude smaller. Although recombination radiation (per event), can be a fraction of the bremsstrahlung power at low electron temperature, recombination of magnetized electron is greatly suppressed. Thus bremsstrahlung is the dominant radiation power loss mechanism.

Even though a closed loop D-shaped solenoidal magnetized plasma target will have to be longer by over 2.5 times, i.e. $(1+\pi / 2)$ longer than an open ended solenoid, total power loss per meter of target is still smaller for closed loop D-shaped solenoidal magnetized plasma; $(1+\pi / 2) \times\left(0.6 \mathrm{~T}+0.77 \mathrm{~T}^{1 / 2}\right)$ versus $4.32 \mathrm{~T}+0.77 \mathrm{~T}^{1 / 2}$; at $15 \mathrm{eV}$ it's 31 versus 68 Watt/meter. And, the difference increases with increasing temperature, especially since the cross-field particle diffusion coefficient will decrease with temperature (to be further examined in the next section). Thus, it is reasonable to pursue the closed loop D-shaped solenoid, which for the achieved parameters, total plasma power lost is $31 \mathrm{Watt} / \mathrm{meter}$.

As computed before from equations 3,5, and 6, each $100 \mathrm{KeV}$ deuteron will deposit $752.4 \mathrm{eV} /$ meter of plasma target. Considering 1 inch-diameter beam and plasma, and 1 Ampere deuterium beam, power deposition (beam power loss) is $752.4 \mathrm{Watt} / \mathrm{meter}$ of tritium plasma (compared to plasma power loss, which is more than an order of magnitude lower). Obviously, significant plasma heating will occur! But, as the plasma heats up and reaches $55 \mathrm{eV}$, cold plasma analysis is no longer valid. As the plasma heats up, its collisionality and collisional particle loss decrease to a point where bremsstrahlung becomes the dominant power loss mechanism. Deuterium power loss rate (and plasma heating) also drops as plasma electrons heat up. Analysis of warm plasma is presented in the next sub-section. 
Worse case scenario is that the deuterium beam generates strong turbulence, which causes particle and energy loss dominated by cross field Bohm diffusion, whose diffusion coefficient is given by ${ }^{30}$

$D_{B}=6.25 \times 10^{6} \mathrm{~TB}^{-1} \mathrm{~cm}^{2} /$ second

and Bohm particle diffusion is given by

$\Gamma=D_{B} \nabla n$ particles $/ \mathrm{cm}^{2} /$ second

and power loss is given by

$P_{B}=\Gamma T \bullet 1.6 \times 10^{-19} \bullet A \mathrm{Watt}$

where $\mathrm{A}$ is the area, across which diffusion takes place. For $\mathrm{T}=15 \mathrm{eV}, \mathrm{B}=380 \mathrm{G}, \mathrm{P}_{\mathrm{B}}$ is $42 \mathrm{KW}$; and the power loss increases with temperature! In this case plasma stays cold. Here as well as elsewhere, it is assumed that energy and particle confinement times are roughly equal (most likely a reasonable assumption in this geometry). Nevertheless, it's obvious that transport in the plasma targets generated in references 33 and 36 was not governed by Bohm diffusion, since their power consumption was two order of magnitude lower than Bohm.

From equation 1, fusion power generated is $3.2 \mathrm{Watt} /$ meter of tritium plasma, which negligible compared to deuterium beam power loss of $752.4 \mathrm{Watt} / \mathrm{meter}$ of tritium plasma. Neutron generation is $1.125 \times 10^{12}$ neutrons/second/meter of tritium plasma, translates to $1.5 \times 10^{9}$ neutrons/second/Watt of beam power loss. This case is two orders of magnitude worse than the gas case with enhanced propagation and an order of magnitude worse than the case without enhanced propagation.

Without plasma heating, this scenario is not promising. Nevertheless there are good reasons to expect significant plasma heating. In addition to experimental evidence that transport in the plasma targets generated in references 33 and 36 was not governed by Bohm diffusion, and that there was no evidence for beam instabilities ${ }^{36}$, theoretically, turbulence is not expected. Deuterium beam ion density is $4.1 \times 10^{9} \mathrm{~cm}^{-3}$, which 5 orders of magnitude lower than the plasma density, is well below any known beam plasma instability thresholds. Furthermore, there is experience with beam instabilities and their stabilization $^{37-39}$.

\section{IVc Deuterium Beam in Warm Electron Tritium Plasma}

Next, the case where the deuterium ions have velocity lower than the electron thermal velocity is explored. From Equation 2, the ion velocity of $3 \times 10^{8} \mathrm{~cm} / \mathrm{sec}$ is matched by electrons whose thermal energy is $54 \mathrm{eV}$, thus requiring an electron temperature larger than $55 \mathrm{eV}$.

When ions are slower than thermal electrons, $v^{\mathrm{i} / \mathrm{e}}\left(\right.$ in $\left.\sec ^{-1}\right)$ is given $\mathrm{by}^{30}$ 
$v^{i / e}=n_{e} Z^{2} \lambda_{i e} 1.6 \times 10^{-9} \mu^{-1} T_{e}^{-3 / 2}$

Deuterium beam power loss rate is given by equation 5, with the equation 13 slowing down rate. From equations 5 and 1 electron temperature, for which "breakeven" condition (fusion energy generated $=$ deuterium ion beam energy loss), can be determined. From 1 and 5,

$\Delta E=Q n \sigma v_{i} \Delta t-v^{i / e} E_{i} \Delta t=0$

since $x=v_{i} \Delta t$. Equation 14 sets breakeven conditions. Substituting for $v^{\mathrm{i} / \mathrm{e}}$ from equation 13 , inserting numerical values for $\mathrm{Q}=17.58 \mathrm{MeV}, \sigma=6 \times 10^{-24} \mathrm{~cm}^{2}$, and $\mathrm{E}_{\mathrm{i}}=100 \mathrm{KeV}$, and solving for $\mathrm{T}_{\mathrm{e}}$ yields "breakeven" for an electron temperature of $861 \mathrm{eV}$. Therefore, breakeven can be achieved for electron temperatures of under $1 \mathrm{KeV}$. If the plasma is preheated above $54 \mathrm{eV}$, no thermalization with tritium ions will occur (runaway condition can prevail).

As it can be seen from equations 13 and 14, breakeven condition depend on the Coulomb Logarithm, which is assume here to be 10 (a typical value used in fusion analysis). At high magnetic fields, that value drops. Hence, breakeven can occur at lower electron temperatures, if large $(\mathrm{T})$ magnetic fields can be used.

Minimum magnetic field required to contain the target plasma can be estimated from $\mathrm{P}=$ $\mathrm{nkT}=\mathrm{B}^{2} / 2 \mu_{0}$. For a density of $3 \times 10^{14} \mathrm{~cm}^{-3}$, and temperature of $861 \mathrm{eV}$, magnetic field of $3.2 \mathrm{kG}$ is needed. At a field of $3.5 \mathrm{kG}$, the electron cyclotron frequency is at the fourth harmonic of $2.45 \mathrm{GHz}$ (the frequency used in microwave oven, for which inexpensive large power supplies are available).

Finally, an attempt is made to estimate equilibrium electron temperature due to deuterium ion beam heating. First it is reasonable to assume that heating to $60 \mathrm{eV}$ will easily occur, hence, equation 13 can be used for ion slowing down rate. Second, semi-empirical expression $(1+\pi / 2) \times\left(0.6 \mathrm{~T}+0.77 \mathrm{~T}^{1 / 2}\right)$, used in the previous sub-section, is modified to reflect temperature dependence of particle loss rate. First factor in the expression accounts for the fact that a plasma target, based on a D-shaped solenoid, extends beyond a transmuter or a breeder core. First term in the second bracket represents energy loss due to diffusion [particle loss rate (0.6) multiplied by average particle energy], while the second term is energy loss due to radiation (bremsstrahlung). But, cross-field diffusion depends on temperature and magnetic field strength. Since any D-shaped solenoid will have a very large aspect ratio, the system can be approximated to a straight solenoid (without end losses), to which classical scaling applies (i.e. neo-classical diffusion does not apply even in the curved section). Diffusion coefficient is step-size square time collision frequency. The first is basically gyro-radius square, which is proportional to $\mathrm{T} / \mathrm{B}^{2}$, while the collision frequency is proportional to $\mathrm{T}^{-3 / 2}$. Since ${ }^{33} \mathrm{~B}=380 \mathrm{G}$, energy loss due to diffusion is $0.6 \mathrm{x}(380 / \mathrm{B})^{2} \mathrm{x}(15 / \mathrm{T})^{1 / 2} \mathrm{xT}$. Hence, plasma power loss rate becomes $3.355 \times 10^{5} \times \mathrm{T}^{1 / 2} / \mathrm{B}^{2}$ Watt/meter ( $\mathrm{T}$ in $\mathrm{eV}, \mathrm{B}$ in Gauss). A simple calculation reveals that 
this value is very close to theoretical classical diffusion, but the use of a semi-empirical is preferable. Total plasma power loss is, to a first approximation, sum of plasma power loss through particle diffusion and power loss due to radiation (bremsstrahlung) from equation 9. Equilibrium electron temperature can be found from the balance of deuterium beam power deposition (from equations 5 and 13, for $1 \mathrm{~A}$ beam) and total plasma power loss.

$3.355 \times 10^{5} \frac{T^{1 / 2}}{B^{2}}+0.77 T_{e}^{1 / 2}=v^{i / e} E_{i} \Delta t=7.92 \times 10^{4} T_{e}^{-3 / 2}$

Solving equation 15 for a magnetic field of about $1 \mathrm{kG}$, yields $\mathrm{T}_{\mathrm{e}}=268 \mathrm{eV}$, i.e. no chance to reach "breakeven" conditions even if the magnetic field is raised to $3.5 \mathrm{kG}$ (electron temperature will reach only $315 \mathrm{eV}$ ). Basically at higher temperatures, bremsstrahlung becomes the dominant energy loss mechanism like in most fusion devices. Actual electron temperature will be lower, since some of the energy will be transferred to the tritium ions.

At an electron temperature of 268 , beam power loss is about 18 Watt/meter, hence efficiency of neutron generation is $6.2 \times 10^{10}$ neutrons/sec/Watt (recall neutron generation is $1.125 \times 10^{12}$ neutrons/second/meter of tritium plasma), which is comparable to the spallation neutron source option. At $\mathrm{T}_{\mathrm{e}}=315 \mathrm{eV}(\mathrm{B}=3.5 \mathrm{kG})$, the efficiency of neutron generation rises to $8 \times 10^{10}$ neutrons/sec/Watt.

\section{Discussion}

Although neutron efficiency generation comparison has been made with the spallation source, used in the GA concept ${ }^{5}$ yielding 40 neutrons per $1 \mathrm{GeV}$ proton, it is important to note that the SNS, which is a pulsed device $(695 \mathrm{~ns}$ at $60 \mathrm{~Hz})$, yields 25 neutrons per 1 $\mathrm{GeV}$ proton $^{40}$ from a mercury target. Only tungsten or uranium targets ${ }^{40}$, which cannot be effectively cooled, can yield 40 neutrons per $1 \mathrm{GeV}$ proton. Furthermore, the European accelerator driven system (ADS $)^{41}$ is based on a $350 \mathrm{MeV}$ proton beam and a liquid $\mathrm{Pb}$ $\mathrm{Bi}$ target yielding 6 neutrons per proton, which is equivalent to 17 neutrons per $1 \mathrm{GeV}$ proton, i.e., a factor of about 3 lower yield than that of the GA concept.

Conversely in the D-T system analysis no neutron multiplication was considered. But, since the fusion products include $14 \mathrm{MeV}$ neutrons, a factor of $2-3$ increase in neutron output is possible. Plasma targets can be put in stainless steel tubes, which are covered with Be or Mo (TCM), which are known to absorb $14 \mathrm{MeV}$ neutrons and release $2-3$ lower energy neutrons. This effect can enhance "neutrons/sec/Watt" and total neutron production of any of the analyzed schemes by a significant factor.

Reaching warm plasma conditions require electron temperatures, which are about a factor of four higher than those of typical hollow cathode arcs. One distinct possibility is to rely on the deuterium beam to heat the plasma, since at low plasma temperatures, beam power deposition is very high. An other possible scenario for achieving such temperatures is to start with a low density plasma target, heat the electrons with microwave to about $60 \mathrm{eV}$ 
(at low density runaway conditions are easily achievable), and increase the plasma density once electron temperature exceeds runaway condition. In this scenario, the plasma ions will stay cold (reducing magnetic field confinement needs). In either case, the ion beam will heat the plasma electrons until bremsstrahlung and other losses prevent further increase in electron temperature. In absence of turbulence or other anomalous loss mechanism, the saturation electron temperature could be about $250 \mathrm{eV}$.

Although power efficiency for generating neutrons described in the above scheme compares favorably with methods based on spallation neutron sources, total neutron generation is much lower than by spallation i.e. $4 \times 10^{15}$ neutrons/second from a dense gas tube and $1.125 \times 10^{12}$ neutrons/second/meter of tritium plasma versus $3.75 \times 10^{18}$ neutrons/sec, which the GA concept is designed to generate. Neutron multiplication will increase neutron flux by a factor of $2-3$. Multiple gas tubes with neutron multiplication could bring total neutron production close to that of spallation. But the pure plasma target option requires too many multiple tubes to be viable. In principle, closed loop D-shaped plasma can be sustained, if gas, droplets, and /or pellets are introduced in a localized region of the straight section, which in sufficient density could result in virtual anodes.

Jets of intense supersonic tritium gas, clusters, droplets, or even frozen pellets shot across the deuterium ion beam could be designed to have optimal line density for deuterium beam slowing down from 125 to $75 \mathrm{KeV}$, though beam heating complicate any calculation for estimating equilibrium conditions. Figures 8,9 , and 10 are schematics of possible internal target embodiments. Ion cooling via charge exchange caused by gas jet might be beneficial in further reducing heat transfer from plasma electrons. Experimental testing of any target system is imperative. Those are intriguing possibilities, which could have some technical challenges, though cost and complexity are minor compared to a spallation neutron source. Nevertheless at this point, it is not clear that total neutron generation needs to match that of the GA concept, which is essentially a point source, while this scheme is basically a well distributed neutron source. Reactor and/or transmuter analysis, based on this novel concept, is needed.

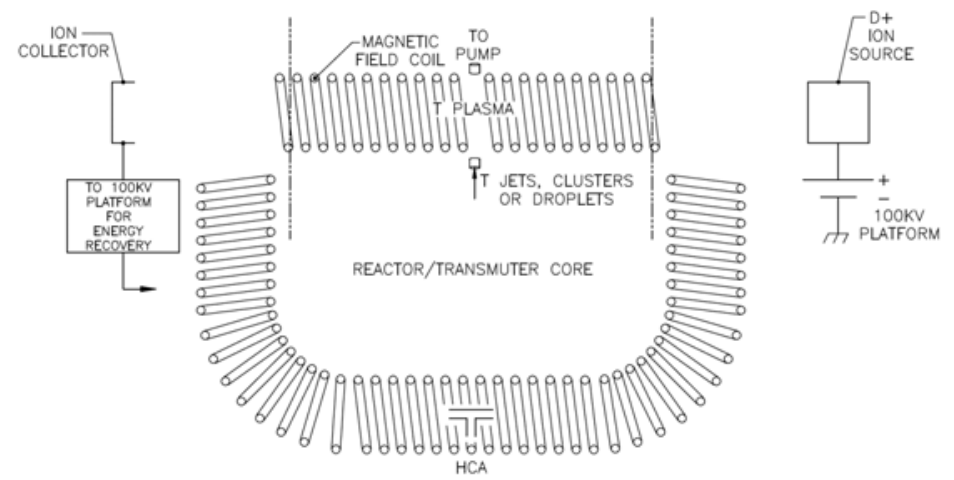

Figure 8 embodiment of figure 6 scheme with an internal target. 


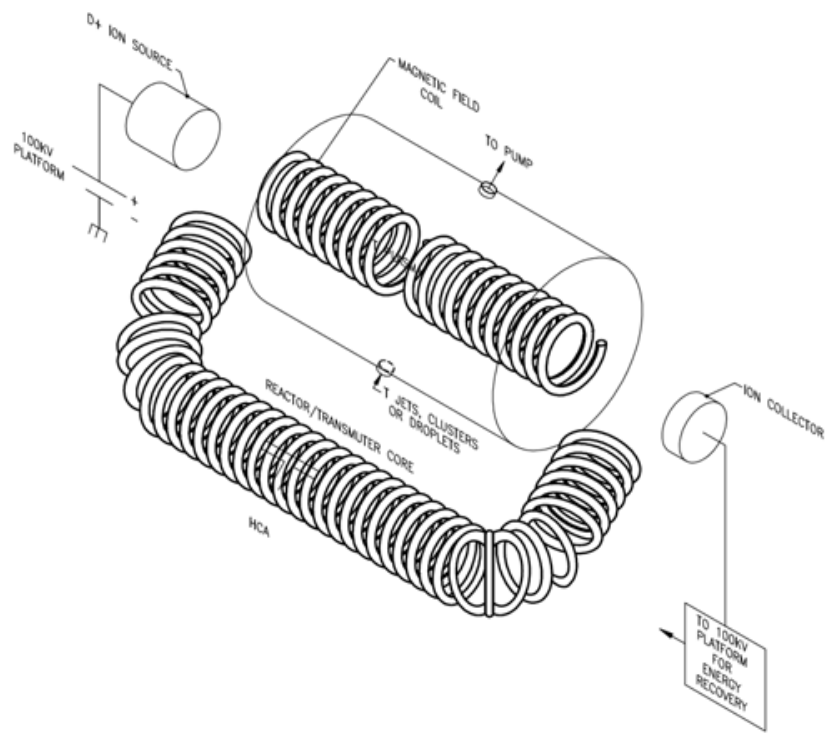

Figure 93 -D display of figure 8 embodiment.

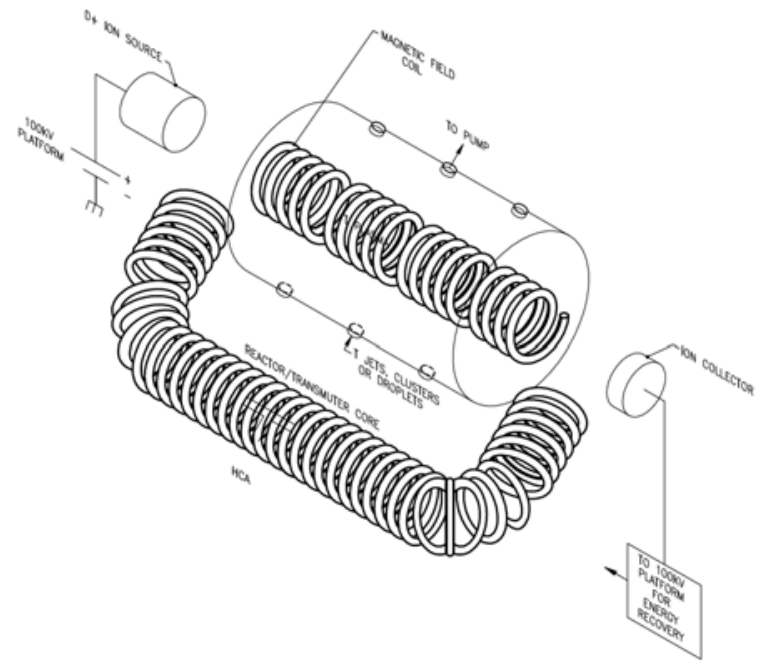

Figure 10 embodiment of figure 9 scheme with multiple internal targets.

In summary deuterium beams injected through tubes of tritium plasma targets (and if needed, with internal tritium gas, droplets, and/or pellets) could be a viable neutron source option for a radioactive waste transmuter or a sub-critical thorium breeder.

\section{REFERENCES}

1. A.R. Tumanyan and A.G. Khudaverdyan, Atomnaya Énergiya, $\underline{79}, 74$ (1995).

2. Carlo Rubbia, "Energy amplifier for nuclear energy production driven by a particle beam accelerator", US Patent 5,774,514 June 30, 1998.

3. Robert Klapisch, "Accelerator driven systems: an application of proton accelerators to nuclear power industry", Europhysics News (2000) Vol. 31 No. 6

4. D.G. Koshkarev, Zhurnal Tekhnichesko, 74, 137 (2004). 
5. C. Rodriguez and A. Baxter, $9^{\text {th }}$ International Conference on Nuclear Engineering, ICONE 9, April 2001, Nice, France, pp 1-11 (2001).

6. L.M. Lidsky, Nuclear Fusion $\underline{15}$, 151 (1975).

7. D.J. Rose and M. Clark Jr., Plasmas and Controlled Fusion, MIT Press 1961.

8. H.A. Bethe, Ann. Physik 5, 325 (1930); H.A. Bethe, Z. Physik 76, 293 (1932).

9. Review of Particle Physics, Journal of Physics G, 33 (July 2006); H.A. Bethe, Phys. Rev. 89, 1256 (1953).

10. The NIST website http://physics.nist.gov/PhysRefData/Star/Text/

11. J.M. Dawson, H.P. Furth, and F.H. Tenney, PRL 26, 1156 (1971).

12. R.F. Post, T.K. Fowler, J. Killeen, and A.A. Mirin PRL 31, 280 (1973).

13. H.P. Furth and D.L. Jassby, PRL 32, 1176 (1976).

14. A. Birnboim, E. Greenspan, and D. Shvarts, Nuclear Fusion 19, 1605 (1979).

15. M. Lampe, PAC'87 March 16-19, 1987 Washington, DC; Proceedings of the 1987 Particle Accelerator Conference, Edited by L. Lindstrom and L. Taylor, p. 1965, IEEE Catalogue Number 87CH2387-9 (1987).

16. F.C. Young et al, PRL $\underline{70}, 2573$ (1993).

17. J. Olsen, D. Johnson, and R. Leeper, Appl. Phys. Lett. 36, 808 (1980).

18. J. Olsen and R. Leeper, J. Appl. Phys. 53, 3397 (1982).

19. P. Ottinger, et al., Proceedings of IEEE, $\underline{80}, 1010$ (1992).

20. Ady Hershcovitch, NIMB 241, 854 (2005).

21. Ady Hershcovitch, Physics of Plasmas 12, 057102 (2005).

22. A. Hershcovitch, J. Appl. Phys. 78, 5283 (1995).

23. W. Gerber, R.C. Lanza, A. Hershcovitch, P. Stefan, C. Castle, E. Johnson, "The Plasma Porthole: a Windowless Vacuum-Pressure Interface With Various Accelerator Applications" at 15th International Conference on the Application of Accelerators, in Research and Industry CAARI'98, Denton, Texas, November 4-7, 1998. AIP Proceedings 475, p. 932.

24. R.C. Lanza, E. Empey, W. Gerber, A. Hershcovitch, P. Stefan, C. Castle, E. Johnson, "High Intensity Neutron Sources with Windowless Gas Target Using a Plasma Porthole" at 3rd International Topical Meeting on Nuclear Application of Accelerators Technology, Long Beach, California, November 14-18, 1999, p. 108 in Proceedings.

25. A. Hershcovitch, Physics of Plasmas, $\underline{5}, 2130$ (1998).

26. D. Salerno, P.T. Pinkoski, A. Hershcovitch, and E. Johnson, NIM A469, 13 (2001).

27. A. de Beer, A. Hershcovitch, C. B. Franklyn, S. van Straaten, and J. Guzek, NIM B170, 259 (2000).

28. A. Hershcovitch, Physics of Plasmas, 15, 057101 (2008).

29. N. Rostoker and M.N. Rosenbluth, Physics of Fluids $\underline{3}, 1$ (1960).

30. Trubnikov, Reviews of Plasma Physics, Vol. 1, (Consultants Bureau, New York, 1965), pp. 105-204; NRL formulary http://wwwppd.nrl.navy.mil/nrlformulary/

31. L. Lidsky et al J. Appl. Phys. 33, 2490 (1962).

32. D. Goebel and T. Forrester NIM 53, 10 (1982).

33. A. Hershcovitch and V.J. Kovarik, Rev. Sci. Instrum. 54, 328 (1983).

34. I. Hershcovitch, V.J. Kovarik, and K. Prelec, NIM 57, 827 (1986).

35. A.I. Hershcovitch, V.J. Kovarik, and K. Prelec, J. Appl. Phys. 67, 671 (1990). 
36. A.I. Hershcovitch, B.M. Johnson, V.J. Kovarik, M. Meron, K.W. Jones, K. Prelec, and L.R. Grisham, Rev. Sci. Instrum. 55, 1744 (1984).

37. A. Hershcovitch and P.A. Politzer, PRL $\underline{36}, 1365$ (1976).

38. A. Hershcovitch and P.A. Politzer, the Physics of Fluids $\underline{22}, 249$ (1979).

39. A. Hershcovitch and P.A. Politzer, the Physics of Fluids $\underline{22}, 1497$ (1979).

40. Ian Anderson, private communication (2009).

41. H. Abderrahim and P. D'Hondt, J. of Nuclear Science and Technology 444, 491 (2007). 